\title{
Enhancing rheumatology education during the COVID-19 pandemic
}

\author{
Saira Bilal ${ }^{1}\left[\right.$. Victoria K. Shanmugam ${ }^{1}[$
}

Received: 23 October 2020 / Accepted: 3 December 2020 / Published online: 27 January 2021

(c) The Author(s), under exclusive licence to Springer-Verlag GmbH, DE part of Springer Nature 2021

\begin{abstract}
During the COVID-19 pandemic, rheumatology educational programs around the world, face the daunting challenge of maintaining education for their trainees. Reduced in-person clinic exposures and social distancing requirements have significantly affected trainee education. Similar to programs around the USA, in early March 2020, our program was faced with an urgent need to pivot both our clinical and educational programs to virtual platforms. Within these limitations, we harnessed innovative educational models and restructured our curriculum to ensure adequate clinical and didactic exposure. We divided trainee's clinical rotations into four blocks, which include Inpatient consult service, Outpatient in-person and procedure clinics, Telehealth Clinics and Research/Elective week. By assigning specific rotations, we were able to ensure fellows were seeing adequate numbers of patients both through telemedicine and inperson while ensuring we complied with social distancing requirements. We further were able to ensure that trainee hands-on procedure training was not compromised. Acknowledging challenges presented by the COVID-19 pandemic and learner engagement in virtual environment, we designed an innovative educational portfolio. Utilizing synchronous and asynchronous learning methods, we have developed multiple complementary educational initiatives including: Rocket Rheumatology, Board Games, At the Elbow, Radiology Reading Rheum, Ultrasound Buddies, The History Rheum, and Rapid-Fire Journal Club. Virtual learning methods will become a cornerstone of medical education moving forwards. The GW Division of Rheumatology has rapidly incorporated innovative educational tools into our curriculum. Our approach will help Rheumatology training programs across the globe enhance rheumatology training.
\end{abstract}

Keywords Education $\cdot$ COVID-19 pandemic $\cdot$ Innovation $\cdot$ Telemedicine

\section{Introduction}

In early 2020, the COVID-19 pandemic spread across the globe and throughout the United States [1]. Introduction of social distancing guidelines [2] and widespread cancellations of in-person conferences significantly affected trainee education [3]. With the ongoing pandemic, educators had to quickly adapt and create new educational curriculum [4]. The purpose of this manuscript is to outline the innovative educational methodologies that George Washington University, rheumatology division has adopted to meet the needs of

Saira Bilal

sbilal@mfa.gwu.edu

Victoria K. Shanmugam

vshanmugam@mfa.gwu.edu

1 Department of Rheumatology, George Washington

University, School of Medicine and Health Sciences, $2300 \mathrm{M}$

street, NW, Washington, DC 20037, USA trainees during the COVID-19 pandemic while minimizing potential exposures and risks. Our priority was to devise novel mechanisms to maintain fellow's education during this time and to optimize the new clinical environment while also providing continuity and at the elbow training to our fellows while ensuring their safety. Our goal was to ensure we maximize face-to-face clinical educational opportunities, enhance educational conferences and guarantee trainees continued improvement of procedural skills including musculoskeletal ultrasound.

We will review the scope of the challenges, and the interventions we adopted to address both the clinical learning environment and also the delivery of didactic education. 


\section{Scope of the challenges}

In accordance with CDC recommendations [5], most US fellowship programs have transitioned to delivering some portion of their clinical care via telemedicine. Many faceto-face educational conferences were cancelled. Similar to other programs around the US, in early March 2020, our program was faced with an urgent need to pivot both our clinical and educational programs to a virtual platform but yet to maintain clinical exposures that are vital to rheumatology training.

\section{Clinical learning environment interventions}

In mid-March 2020, it became clear that we needed to limit our clinical exposures to minimize risk to our fellows and faculty and to preserve personal protective equipment in case the hospital system became overwhelmed. In accordance with CDC guidelines [5], we rapidly adopted a telemedicine platform for delivery of ongoing care to stable patients with chronic rheumatic diseases. The GW Section of Innovative Practice and Telehealth trained faculty over a weekend [6] and the first visits were scheduled March 16, 2020. We were the first division within our institution to utilize the telemedicine platform [7]. However, as we made this transition, the primary focus was to maintain clinical operations and trainee education was secondary.

\section{Clinical learning interventions}

As the pandemic rapidly evolved, the Accreditation Council for Graduate Medical Education (ACGME) accelerated implementation of telemedicine requirements, and effective March 18, 2020 fellows and residents were permitted to participate in use of telemedicine to continue patient care. The George Washington University School of Medicine and Health Sciences Graduate Medical Education Committee developed a policy for the involvement of trainees in telemedicine and upon approval our trainees were then able to participate in outpatient telemedicine visits. With this change, we restructured rotations to allow the trainees to focus their time appropriately during each learning environment. On a weekly rotating schedule, the trainees rotate between the following assignments:

\section{Inpatient consult service}

Trainee covers GW hospital consult service. Most inpatient consults are still covered using face-to-face visits with appropriate personal protective equipment. However, during periods when the hospital was at surge capacity, the inpatient-consult service was approved to utilize telemedicine to provide consultation via Zoom with the patients being provided bedside Health Insurance Portability and Accountability Act (HIPPA) compliant hospital devices. In this format, faculty and fellow connected with the patient via Zoom, obtain history and physical exam, and discuss treatment plan with the patient. When necessary, the team would also do a bedside physical exam on a case-by-case basis. We found this to be an extremely beneficial mechanism to deliver consults since it allowed us to provide timely consultation to the Emergency Room and Surgical Teams. We continue to conduct the majority of our consults with inperson consultation, but are determining on a case-by-case basis when to utilize telemedicine for inpatient consults, and overall, we do think it is a beneficial addition to our training program moving forwards.

\section{Outpatient in-person clinic and procedure clinics}

Trainee covers all outpatient and procedure clinics with a pre-defined assignment according to the attending in clinic each day such that there is consistency and continuity. Trainee covers all procedure and musculoskeletal ultrasound clinics. The purpose of this change was to account for reduced clinic exposures due to the majority of clinics now being conducted via telehealth. To ensure that our fellows, continue to learn procedures, and how to complete rheumatologic physical exams joint aspirations and injections, we developed a schedule that would allow the fellow on the outpatient week to attend a total of 9 clinics including 3 procedure clinics. This ensures that they have access to the varied and high-yield cases who are seen in the clinic (many of whom have very important clinical findings) and that they can participate in any procedures. Within the first few weeks of this new clinic structure, fellows noted a significant uptick in their procedure logs.

\section{Telehealth clinics}

Trainee covers specific telehealth clinics with a consistent assignment so that they continue to achieve consistency and continuity in patient care, and such that they can be exposed to the breadth and depth of rheumatologic care provided by different faculty within the division. Fellows are assigned specific cases prior to the scheduled clinic and if necessary, discuss the case with the supervising attending prior to the appointment. The Fellow and faculty join the Zoom telehealth visit simultaneously with the patient. With the patient's consent, the fellow then leads the visit, acquires the history and virtual physical examination, and develops an assessment and plan. The observing attending then asks any additional questions and discusses the case with the fellow, while engaging the patient in the discussion so that the 
patient understands the diagnosis and plan. Direct faculty supervision throughout the visit allows us a unique opportunity to evaluate the trainee's history taking and clinical skills as well as their understanding of disease process and counselling techniques. This also allows faculty to observe fellow's communication and time management skills. Upon completion of the visit, faculty are available to discuss the case further, provide feedback to the fellow and address any remaining concerns. Often this debrief occurs at the end of the clinic day. We have received overwhelmingly positive reviews of the telemedicine platform and both fellows and faculty feel it has been an incredibly beneficial addition to the training program.

\section{Research/elective week}

Trainees are provided with dedicated time to complete research projects, quality improvement projects and to rotate through electives, such as learning how to read bone density scans. This also allows us to have some flexibility if a fellow requires quarantine due to COVID-19 exposure.

As the COVID-19 pandemic continues to impact medical education, we have embraced this opportunity to train our fellows in telemedicine and to provide ongoing patient care during these challenging times [8], while recognizing the vulnerabilities of these platforms including technologic failures and handling of sensitive patient information [9].

\section{Didactic education interventions}

Prior to the COVID-19 pandemic, our conference and training portfolio included journal club, case conferences, grand rounds, patient safety conference and research conference. We also recognized that much of our teaching occurred at the bedside and during clinical consult rounds. During the rapid redesign of our program, we reflected on the modalities we had previously relied on and deliberately assessed how we could reincorporate them into our curricular redesign. We realized that many of our most valuable teachable moments occurred in informal settings. Trainees had previously benefitted from bedside hands-on expert advice on how to dose and administer rheumatologic therapies, and nuggets of rheumatology history as we drew their attention to seminal manuscripts or pivotal moments in rheumatology history in which treatment and diagnostic paradigms changed. Although clinical interactions were still occurring, they were often shorter with a major focus on administering clinical care as safely as possible. As we approached the re-design of our educational portfolio, we wanted to incorporate these types of learning opportunities.

Another acknowledged challenge presented by the COVID-19 pandemic was learner engagement. As we considered different educational modalities, we wanted to incorporate both the learner driven models adopted by many massive open online (MOOC) courses [10] that allow learners to watch on demand and as their educational needs evolve, but also to include engagement strategies, such as gamification tools, to allow learners to engage with the materials. Finally, we prioritized providing trainees with opportunities to interact with faculty [11].

Building upon the established learning pyramid from National Training Laboratories (NTL) (Fig. 1), we sought to design an innovative educational portfolio for our Rheumatology trainees which would be administered through a Zoom conferencing platform that we were already using for clinical tele-medicine visits. Utilizing a hybrid of synchronous and asynchronous tools, we designed educational initiatives to complement our previous didactic portfolio [12].

\section{Rocket rheumatology}

Rocket rheumatology is intended to be an asynchronous learning opportunity. In the first few weeks as we rolled out the program, it was delivered synchronously to ensure we had a format that resonated with our trainees. A single faculty member presents a short (10-15 $\mathrm{min})$ recorded didactic focused on a very specific topic within rheumatology with appropriate slides. We have since then utilized the same platform to record our usual fellows' introductory series, and our Rheumatology educational lectures for the musculoskeletal module delivered to the second-year medical students. We will continue to build upon the library of recorded lectures ranging in breadth and depth from basic level (for medical

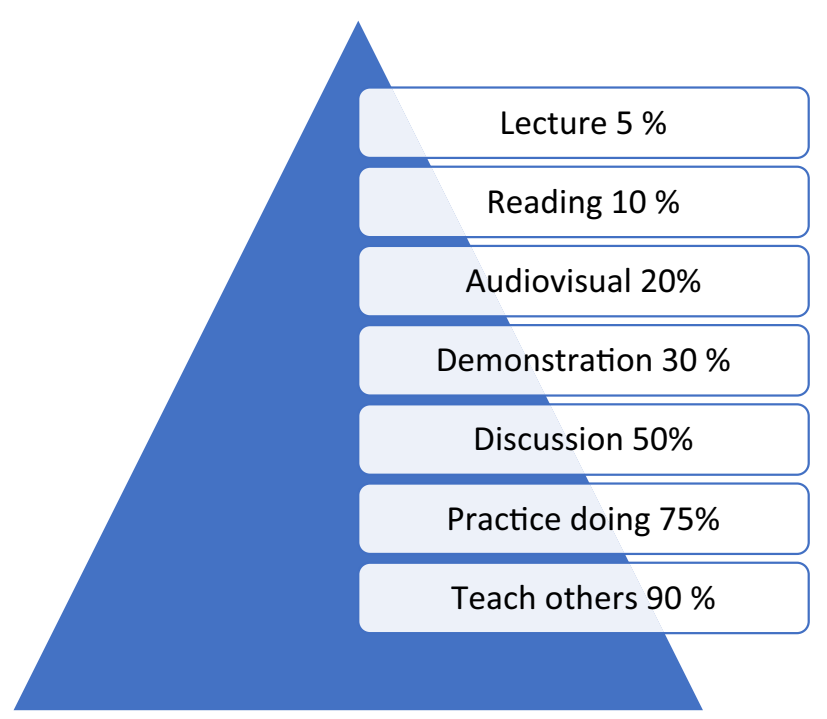

Fig. 1 The learning pyramid 
students) to advanced (aimed at the level of rheumatology fellows). Each Rocket Rheumatology session is peerreviewed by two members of the Rheumatology Faculty to ensure the content meets the intended learning objectives. Trainees watch these videos prior to the synchronous learning occurring during our weekly fellow's conference.

\section{Board games}

This synchronous educational component occurs at the start of our fellow's conference each week. We review ABIM board-style questions pertinent to the Rocket-rheumatology topic assigned for the week. These opportunities allow the fellows to work through board-style problems with faculty providing advice and mentorship. We have broadened this portfolio harnessing different gamification strategies including anatomy games, such as bone bingo, and a team-based knowledge bowl game, which supports synchronous learning while minimizing virtual learning fatigue.

\section{At the elbow}

This synchronous learning opportunity occurs during our fellow's educational conference time. Harnessing the breakout room function on $\mathrm{Zoom}^{\odot}$, we allow the fellows to role-play counselling a standardized patient (one of the faculty members) how to initiate specific medications. This simulates the "at the elbow education" which would have previously occurred in the clinic workroom. We review drug selection and contraindications, potential risks and side effects as well as required monitoring. Another observing faculty member provides feedback and re-instruction as necessary. Faculty weigh in on individual stylistic factors on how they utilize specific medications. This active-learning method allows the fellows to really engage and think about how they counsel patients and provides them an opportunity to hone their counselling skills in the telehealth environment without the time pressures of the clinic visit $[12,13]$. Faculty provides feedback at the time of the encounter while the fellow is still in the break room so that any knowledge gaps can be addressed in real time and to provide a safe and non-threatening environment for the learner to receive this training.

\section{Radiology reading rheum}

This synchronous learning opportunity is being introduced monthly to allow fellows to interact with our musculoskeletal radiology faculty. Rheumatology Faculty submit cases ahead of the conference and the fellows then "read" the films with guidance and direction from the rheumatology and radiology faculty. This interactive session allows fellows to hone their diagnostic and interpretative skills.

\section{Ultrasound buddies}

Previously our institution had hosted joint injection and ultrasound workshops for fellows four times per year. However, due to social distancing requirements, these workshops could no longer be conducted in the same format. To ensure continued exposure to ultrasound, we have developed a buddy system in which our trainees buddy up with RhMSUS certified faculty member such that each mentor has with one junior and one senior mentee. For the first six months, training is focused on allowing the fellows to scan normal joints. In each month, the mentor assigns a specific anatomic region for study and self-scanning, and provides an example video. The fellows are assigned dedicated time in the ultrasound room so they can utilize the machine to scan themselves at a time when patients are not in the clinic. Using the treasure hunt method of gamification, they are given a list of specific views or images to capture. Upon completion of the monthly assignment, they receive peer feedback on their image acquisition from both their co-fellow and faculty mentor buddies.

\section{The history rheum}

Purpose of the history rheum sessions is to ensure fellows continue to learn about seminal papers and moments in rheumatology history. Fellows pair up with a faculty member to review historical background pertaining to landmark discoveries in the field of rheumatology and record a short interview of their discussion. We envision these interviews stimulating our fellows to read some of the seminal papers in our field and to reflect on how rheumatology has evolved over the years.

\section{Rapid-fire journal club}

We have redesigned our journal club to focus on topic areas of rheumatology. Each of our four fellows is assigned an article that the supervising faculty have determined is seminal in the field. The fellow prepares a short, 10-min presentation dissecting the study design and results. Fellows then answer rapid-fire questions from faculty and co-fellows pertaining to the study design and interpretation.

\section{Intra-city clinico-pathological conference}

Prior to COVID-19, we had collaborated with the other DC Fellowship Training Programs with a monthly CPC style conference. We have continued this conference but moved to the Zoom ${ }^{\odot}$ platform and are teaching our fellows skills on presentation in this virtual format, similar format as adopted by some other programs [14]. 
Fig. 2 Diagram of goals and action plan for enhancement of rheumatology training during COVID-19 pandemic
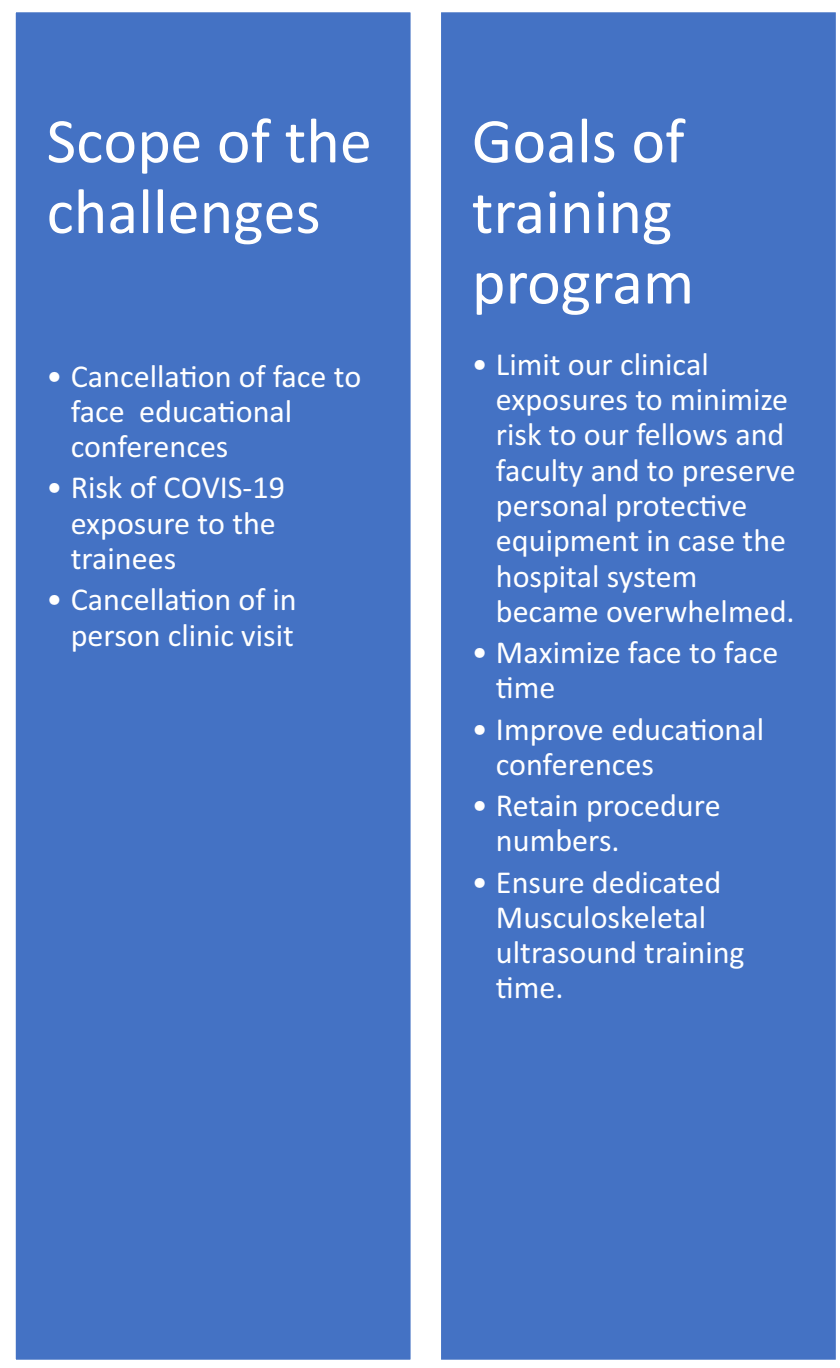

\section{Conclusion}

The COVID-19 pandemic has revolutionized the practice of clinical medicine in the United States and around the world [15] and created unique and complex challenges for ongoing rheumatologic education. The EULAR Working Group on Training has identified fundamental needs while pivoting towards virtual leaning, which include the need for frequent feedback to trainees, adequate time for quality observation, incorporating teamwork and professionalism into assessment systems and developing approaches in close proximity to clinical practices [16].

Within our rheumatology program, we quickly embraced and adapted to this new style of practicing medicine and delivering education. Moving forward, it is clear that virtual learning methods will become a cornerstone of medical education and the interventions we adopted are likely to be scaleable to other programs (Fig. 2). As this portfolio expands, we will incorporate learner feedback and develop evaluation tools to assess efficacy of these various modalities. We believe that our approach will help Rheumatology training programs across the globe to incorporate these methods to enhance trainees learning experience.

\section{Compliance with ethical standards}

Conflict of interest Saira Bilal has nothing to disclose. Victoria K. Shanmugam has nothing to disclose.

\section{References}

1. Ahn D-G et al (2020) Current status of epidemiology, diagnosis, therapeutics, and vaccines for novel coronavirus disease 2019. 
J Microbiol Biotechnol 30(3):313-324. https://doi.org/10.4014/ jmb.2003.03011

2. World Health Organization (2020) Coronavirus disease (COVID19) advice for the public. https://www.who.int/emergencies/disea ses/novel-coronavirus-2019/advice-for-public. Accessed 15 Nov 2020

3. Ferrel $\mathrm{M}$ et al (2020) The impact of COVID-19 on medical education. Cureus 12(3):e7492. https://doi.org/10.7759/cureus.7492

4. Lucy C et al (2020) The transformational effects of COVID-19 on medical education. J Am Med Assoc 324(11):1033-1034. https:// doi.org/10.1001/jama.2020.14136

5. Centers for Disease Control and Infection (2020), Framework for healthcare systems providing non-COVID-19 Clinical care during the COVID-19 pandemic. https://www.cdc.gov/coronaviru s/2019-ncov/hcp/framework-non-COVID-care.html. Accessed 15 Nov 2020

6. Sikka N (2020) Innovative practice and telehealth. https://smhs. gwu.edu/emed/interest-sections/innovative-practice-and-teleh ealth. Accessed 15 Nov 2020

7. Ward IM et al (2016) How critical is tele-medicine to the rheumatology workforce? Arthritis Care Res. https://doi.org/10.1002/ acr.22853

8. Pepe D et al (2019) Involving physicians-in-training in the care of patients during epidemics. J Grad Med Educ 11(6):632-634. https://doi.org/10.4300/JGME-D-19-00354.1

9. Goel A et al (2020) Social media in the times of covid-19. J Clin Rheumatol 26(6):220-223. https://doi.org/10.1097/RHU.00000 00000001508

10. Sinha $\mathrm{M}$ et al (2020) Human touch in digital education-a solution. Mehul Clin Rheumatol 39:3897-3898. https://doi. org/10.1007/s10067-020-05448-y
11. Ward $\mathbf{J}$ et al (2001) Communication and information technology in medical education. Lancet 357(9258):792-796. https://doi. org/10.1016/S0140-6736(00)04173-8

12. Parslow GR (2012) Commentary: synchronous and asynchronous learning. Biochem Mol Biol Educ 40(3):212. https://doi. org/10.1002/bmb.20610

13. Kinney $\mathrm{M}$ et al (2010) The use of self and role play in social work education. Mental Health Training Edu Pract 5(4):27-33. https:// doi.org/10.5042/jmhtep.2010.0688

14. Koumpouras F et al (2020) Stand together and deliver: challenges and opportunities for rheumatology education during the COVID19 pandemic. Arthritis Rheumatol 72(7):1064-1066. https://doi. org/10.1002/art.41278

15. Cai K et al (2020) The impact of COVID-19 on rheumatology clinical practice and university teaching in Sydney, Australia. Eur J Rheumatol 7(Suppl 2):S91-S93. https://doi.org/10.5152/eurjr heum.2020.20060

16. Ahmed $\mathrm{S}$ et al (2020) Moving towards online rheumatology education in the era of COVID- 19. Clin Rheumatol 39:3215-3222. https://doi.org/10.1007/s10067-020-05405-9

17. The Learning Pyramid: https://www.educationcorner.com/thelearning-pyramid.html. Accessed 15 Nov 2020

Publisher's Note Springer Nature remains neutral with regard to jurisdictional claims in published maps and institutional affiliations. 